\title{
Poor response to azithromycin in cutaneous leishmaniasis leading to a premature interruption of a multicentric phase III clinical trial in Brazil
}

\author{
Antonio Toledo Junior ${ }^{[1]}$, André Bastos Daher ${ }^{[2],[3]}$, Thaís Alves Amaral ${ }^{[2],}$ \\ Sílvio Fernando Guimarães Carvalho ${ }^{[4]}$, Gustavo Adolfo Sierra Romero ${ }^{[5]}$ \\ and Ana Rabello ${ }^{[6]}$
}

[1]. Serviço Multiprofissional de Infectologia, Universidade José do Rosário Vellano, Belo Horizonte, MG. [2]. Plataforma de Pesquisa Clínica, Vice-Presidência de Pesquisa e Laboratórios de Referência, Fundação Oswaldo Cruz, Rio de Janeiro, RJ. [3]. Departamento de Pesquisa Clínica - Farmanguinhos, Fundação Oswaldo Cruz, Rio de Janeiro, RJ. [4]. Departamento de Doenças Infecciosas, Hospital Universitário Clemente de Faria, Universidade Estadual de Montes Claros, Montes Claros, MG. [5]. Núcleo de Medicina Tropical, Universidade de Brasília, Brasília, DF. [6]. Laboratório de Pesquisa Clínica, Centro de Pesquisas René Rachou, Fundação Oswaldo Cruz, Belo Horizonte, MG.

\begin{abstract}
Introduction: Parenteral antimony-based compounds are still the standard of care for cutaneous leishmaniasis (CL) treatment in many countries, despite their high toxicity. Previous studies showed that oral azithromycin could be an option for CL treatment. The aim of this study was to evaluate efficacy and safety of oral azithromycin (AZ) for CL treatment compared with injectable meglumine antimoniate (MA). Methods: This was a randomized, open-label, 2-arm, non-inferiority clinical trial. Treatmentnaïve patients with localized CL were treated with MA $(15 \mathrm{mg} / \mathrm{kg} /$ day up to $1,215 \mathrm{mg})$ or AZ (500mg/day) during 20 consecutive days. The primary efficacy end point was a CL cure 90 days after treatment completion. The analysis was performed with intention-to-treat (ITT) and per protocol (PP) analyses. After an anticipated interim analysis, the study was interrupted due to the high failure rate in the azithromycin group. Results: Twenty-four volunteers were included in each group. The MA group had a higher cure rate than the AZ group with the ITT and PP analyses, which were $54.2 \%$ versus $20.8 \%$ [relative risk (RR) $1.97 ; 95 \%$ confidence intervals (95\%CI) $1.13-3.42$ ] and $72.2 \%$ versus $23.8 \%$ (RR 3.03; 95\%CI 1.34-6.87), respectively. No unexpected adverse events were observed. Conclusions: Azithromycin is ineffective for CL treatment and does not seem to have a role in the therapeutic arsenal for CL.
\end{abstract}

Keywords: Azithromycin. Cutaneous leishmaniasis. Leishmania braziliensis. Randomized controlled trial. Meglumine antimoniate.

\section{INTRODUCTION}

Cutaneous leishmaniasis (CL) is a serious public health problem, with approximately 0.69 to 1.2 million new cases occurring per year worldwide ${ }^{1}$. Ten countries, including Afghanistan, Algeria, Colombia, Brazil, Iran, Syria, Ethiopia, North Sudan, Costa Rica and Peru, together account for 70 to $75 \%$ of the global estimated CL incidence ${ }^{1}$. In Brazil, from 2008 to 2012 , a mean of 21,736 new cases per year of tegumentary leishmaniasis were reported ${ }^{2}$. CL may manifest as single or multiple skin lesions, which occur in the same region or disseminated in different body regions, and can have a severe impact on the patient's quality of life $\mathrm{e}^{3,4}$.

Address to: Dra. Ana Rabello. Lab. Pesquisa Clínica/CPqRR/FIOCRUZ. Av. Augusto de Lima 1715, 30190-002 Belo Horizonte, MG, Brasil.

Phone/Fax: 5531 3349-7708

e-mail: ana@cpqrr.fiocruz.br

Received 31 October 2014

Accepted 8 December 2014
Cutaneous leishmaniasis treatment accelerates cures, reduces scarring and relapses, and it is expected that it may reduce the frequency of disease dissemination to the upper airway mucosal surfaces. Parenteral antimony is still the standard of care in many countries. However, the once daily intramuscular or intravenous injection over 20 days normally causes discomfort to patients and it is difficult to administer in some poorly resourced countries or regions. Moreover, antimony-based compounds are toxic drugs and may cause serious and life-threatening events, such as acute pancreatitis and cardiac arrhythmias. Frequently, patients have formal contraindications to antimony use, such as uncontrolled diabetes mellitus, liver or heart disease, and chronic kidney disease. These conditions are more common in older patients, appearing after a few days of drug administration and when higher doses are required. Other treatment options include pentamidine and amphotericin B, which are both injectable and have a safety profile that is similar to antimony and the lipid formulations of amphotericin $\mathrm{B}$, wich are expensive optionsfor the majority of the CL endemic countries. Various studies evaluated oral drugs as candidates for CL treatment, such as miltefosine ${ }^{5-7}$, fluconazole ${ }^{8,9}$, and azithromycin ${ }^{10-15}$, with controversial results or no clear evidence of efficacy. 
Azithromycin, an azalide antibiotic is largely used to treat bacterial infections, and was identified as a potential option for CL treatment due to its wide tissue distribution and high intracellular concentration, including macrophages ${ }^{16}$. Three studies showed that azithromycin was active against Leishmania parasites. Krolewiecki et $\mathrm{al}^{17}$ showed both in vitro and in vivo activity of azithromycin against Leishmania (Leishmania) major promastigote and amastigote forms ${ }^{17}$. Tanyuksel et al ${ }^{18}$ demonstrated in vitro the potential leishmanicidal effect of azithromycin on intracellular amastigote $L$. $(L$.) major forms in peritoneal macrophages from mice ${ }^{18}$. Lastly, Oliveira-Silva et a ${ }^{16}$. showed that azithromycin was active in vitro against Leishmania (Leishmania) amazonensis, Leishmania (Viannia) braziliensis, and Leishmania (Leishmania) chagasi and suggested that the drug might offer an alternative to current leishmaniasis treatments ${ }^{16}$.

Despite these promising in vitro results, clinical studies of azithromycin and CL had controversial results. Prata et al. ${ }^{13}$ showed a cure rate of $85 \%$ in an open non-controlled trial with 20 patients $^{13}$. Four studies in Brazil showed low efficacy of azithromycin when compared with meglumine antimoniate ${ }^{10,14,15}$. Two others studies evaluated azithromycin against Old World leishmaniasis, both with poor responses ${ }^{11,12}$. These studies enrolled a small number of volunteers, 22 to 49, with varied clinical protocols, restraining a definitive conclusion regarding the possible role of azithromycin as a therapeutic option for CL. The aim of this study was to evaluate the efficacy and safety of oral azithromycin for CL treatment compared with injectable meglumine antimoniate.

\section{METHODS}

\section{Study population}

Treatment-naïve patients with localized cutaneous leishmaniasis were included in the study after signing the informed consent form if they met the following inclusion criteria: 1) age between 14 to 65 years; and 2) $\leq 6$ apparent cutaneous lesions that were compatible with CL and a positive Montenegro reaction test $(\geq 5 \mathrm{~mm})$, followed by parasitological or molecular confirmation of the CL infection (which included a direct exam, culture, pathological exam, and/or kinetoplast deoxyribonucleic acid (kDNA) detection by polymerase chain reaction). L. (Viannia) braziliensis was the species identified in deoxyribonucleic acid (DNA) samples extracted from biopsy fragments of 29 patients. Leishmania (Viannia) braziliensis is the most common species found in the patients at the study reference center $(>90 \%)$ (unpublished data) and in the study-area $(92.5 \%)^{19}$.

Patients who had or met one of the following criteria were not eligible to participate: disseminated leishmaniasis; the presence of mucosal lesions; pregnancy [confirmed by beta human chorionic gonadotropin ( $\beta$-hCG) in their blood sample]; breast feeding; diseases that interfere with scar healing; primary or acquired immunodeficiency; immunosuppressive drug use; use of any topical or oral medication that could interfere with the healing process or with potential leishmanicidal action, including antibiotics; decompensated chronic diseases; any electrocardiogram (ECG) abnormalities that contraindicated meglumine antimoniate use; use of any medication that could prolong the QTc interval; any diseases or conditions that may lead to non-compliance to protocol, including alcohol abuse; and intolerance to azithromycin, macrolides or meglumine antimoniate.

\section{Study design}

This multicenter, randomized, open-label, 2-arm parallel group, non-inferiority clinical trial was conducted in two leishmaniasis reference centers: The René Rachou Research Centre/Oswaldo Cruz Foundation (CPqRR/FIOCRUZ), and Montes Claros State University (Unimontes). Both centers are located in Minas Gerais, Brazil. Randomization used blocks of six and a 1:1 allocation rate were generated by using the Web site Randomization.com [http://www.randomization. com]. Envelopes that were sequentially numbered, opaque and sealed were provided to the local clinical coordinator. Allocation followed the recruitment sequence, and the patient's name was written on the envelope before it was opened.

Eligible patients were allocated into one of the two treatment arms: Group A (meglumine antimoniate) or group B (azithromycin). The group A patients were treated, according to the Brazilian Ministry of Health recommendation, with $15 \mathrm{mg} /$ $\mathrm{kg}$ /day of intravenous or intramuscular meglumine antimoniate (maximum daily dose of $1,215 \mathrm{mg}$ ) over 20 consecutive days (Glucantime ${ }^{\circledR}$ - Aventis, batch number: 605022). The group B patients were orally treated with one $500 \mathrm{mg}$ azithromycin tablet per day over 20 consecutive days (Zitromax ${ }^{\circledR}$ - GSK, batch numbers 6186401403; 0864009). All of the treatments were performed in an ambulatory setting.

Treatment adherence for both treatment groups was supervised daily by the health professionals of the public ambulatory unit that was closest to each patient's home. Additionally, all of the drug administration was registered in a specific form and signed by the health professional under close supervision of the study team.

Treatment was considered incomplete if it was interrupted before the $16^{\text {th }}$ dose or if the treatment lasted for more than 30 days. A treatment was considered irregular if there was an interval greater than $72 \mathrm{~h}$ between any drug doses.

\section{Safety and efficacy evaluations}

Clinical evaluations, which included vital signs and laboratory tests, (including complete blood cell counts, biochemistry tests, urinalysis, and a 12-lead ECG) were assessed at enrollment, during treatment (days 5, 10, and 20), and 10 days after treatment. A clinical evaluation was also performed at months 1, 2, 3, and 6 after treatment. The lesion areas were measured using a $0.1 \mathrm{~cm}$ precision caliper, and the following formula was applied: D1 x D2 $x \pi / 4$, where D1 and D2 are the greatest and smallest diameters, respectively. Photographs were taken to record the lesion evolution.

To assess safety, clinical and laboratorial adverse events were investigated on an outparient basis. The participants were withdrawn from the study if the patient presented with a severe or life threatening event or if the investigator was concerned about treatment safety. 
The primary efficacy end point was CL cure rate by intention-to-treat (ITT) and per protocol (PP) analyses. A cure was defined as complete lesion healing and re-epithelialization without inflammatory infiltration and erythema until 90 days after the treatment ended. Patients withdrawn from the study due to AEs or loss to follow-up were considered treatment failures in the ITT analysis.

The secondary endpoints were as follows: a delayed CL cure (180 days after treatment), CL recurrence between cure and 180 days after treatment, and the percentage of healed lesions at the end of treatment and at 30,60, and 90 days after the treatment ended. Recurrence was defined by the reappearance of a previous lesion or a new cutaneous or mucosal lesion. Patient lost to follow-up were considered as recurrences in the ITT analysis. All of the secondary efficacy endpoints were analyzed by ITT and per protocol (PP).

The safety analysis included all patients who received at least one dose of study drugs. Clinical, laboratory and ECG abnormalities were categorized according to the acquired immunodeficiency syndrome (AIDS) clinical trial group (ACTG) criteria.

Patients who did not respond to azithromycin were treated with the standard therapy with meglumine antimoniate, the same regime of the group A arm, or second-line drugs, if necessary.

\section{Statistical analysis}

Initially, a sample size of 310 patients in each group was calculated using an accuracy method, with an alpha error $=0.05$ and a power $=0.8$, and considering a $60 \%$ cure rate in both arms. The inferior limit of the $95 \%$ confidence interval (one-sided) between the azithromycin and meglumine antimoniate cure rates should not have been less than $-10 \%$ in order to consider the azithromycin treatment as non-inferior. The final sample size was 682 patients, which took into consideration a possible loss of follow-up period of $10 \%$.

The data were summarized as frequencies and percentages for categorical variables and as the means \pm standard deviation (SD) for continuous variables. Fisher's exact test (two-sided) and a two-sample t-test were used to compare the differences between the groups for the categorical and continuous variables, respectively. P-values lower than 0.05 were considered statistically significant. The statistical analysis was made using the Statistical Package for the Social Sciences (SPSS) Statistics software - version 19 (IBM, US).

Due to the high failure rate in the azithromycin group, the principal investigator, in accordance with the Institutional Sponsor, requested an evaluation of an external ad-hoc Review Board, which recommended a trial interruption. No new inclusions were conducted and the enrolled patients were followed according to the protocol.

\section{Ethical considerations}

The clinical study protocol and informed consent were reviewed and approved by the Rene Rachou Research Centre, Oswaldo Cruz Foundation [Centro de Pesquisa René Rachou, Fundação Oswaldo Cruz (CPqRR, FIOCRUZ)],
(CAAE 0010.0.246.000-06/CEPSH-CPqRR 20/2006) and Montes Claros State University - Unimontes (Comitê de Ética em Pesquisa da Universidade Estadual de Montes Claros 2050) ethics committees. The Brazilian National Council on Ethics in Research (CONEP) accredits these committees. The project has also been approved by the Ethics Review Committee of the University of Brasilia from where a third trial site was expected to be coordinated but was cancelled due to the study interruption. The clinical study was conducted in accordance with the Good Clinical Practice (ICH 1996) and Brazilian National Health Council (CNS) resolutions 196/1996 and 251/1997. The study is registered at www.Clinicaltrials.gov (NCT00682656). Written informed consent was obtained for every subject prior to enrollment. For patients younger than 18 years of age, a written informed consent was also obtained from their legal representative. All of the subjects were informed about the nature of the trial and possible risks bound to it and that they were free to withdraw the consent of participation at any time. The investigators and study staff observed the confidentiality of the records.

\section{RESULTS}

Seventy-seven patients were screened and 48 volunteers with confirmed CL were enrolled between August/2008 and September/2011. These included 43 subjects at Belo Horizonte and five at Montes Claros. The last 6-month follow-up visit was conducted on March 2012.

Table 1 shows the baseline demographic and clinical characteristics. The majority of the subjects were male (38-79.2\%), their ages ranged from 15 to 56 years (mean $34.5 \pm 12.2$ ), and their years of schooling ranged from 2 to 17 years (mean $8.3 \pm 3.7$ ). Almost $2 / 3$ of the patients had only one lesion, and the time since the first lesion occurred ranged from 15 to 425 days (mean 98.6 \pm 67.5 ). Additionally, the lesions of 29 (61.7\%) patients had $\leq 90$ days of progression. The mean lesion area was $5.17 \mathrm{~cm}^{2}(\mathrm{SD}=10.11)$. There were no baseline demographic and clinical characteristic differences between both groups .

Figure 1 shows the participants treatments and their followups. Twenty-four participants were enrolled in each treatment group. Four subjects from the meglumine antimoniate group withdrew during treatment due to adverse events. All of the patients enrolled in the azithromycin group completed their treatment. During the 3 months post-treatment, five patients were lost to follow-up, which included three from the azithromycin group and two from the meglumine antimoniate group. Six patients were initially considered as failures; however, they were cured between 3 and 6 months after their treatments ended. These included two in the azithromycin group and four in the meglumine antimoniate group (Figure 1).

Table 2 shows the primary and secondary efficacy endpoints. Three months after the end of the treatments, the meglumine antimoniate group had a higher cure rate than the azithromycin group in the ITT and PP analyses, (54.2\% versus $20.8 \%$ [relative risks (RR) 1.97; 95\% confidence intervals $(95 \% \mathrm{CI})$ 
TABLE 1 - The baseline demographic and clinical characteristics of each group.

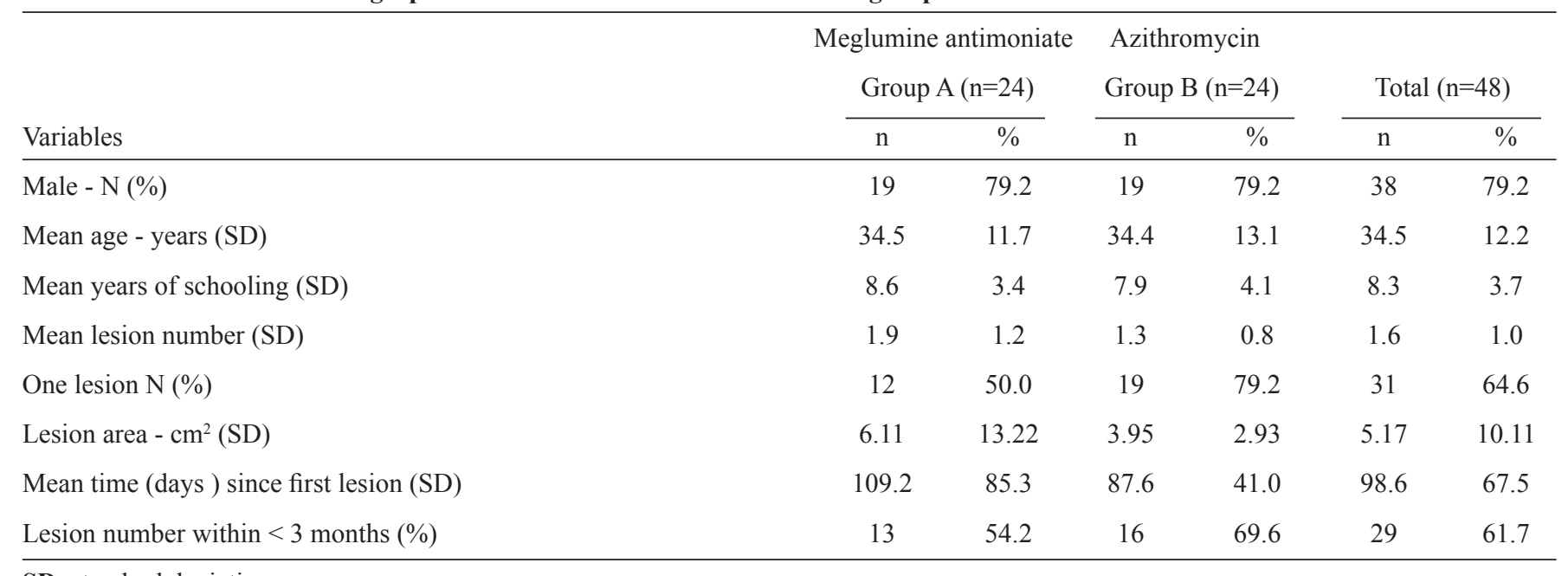

SD: standard deviation.

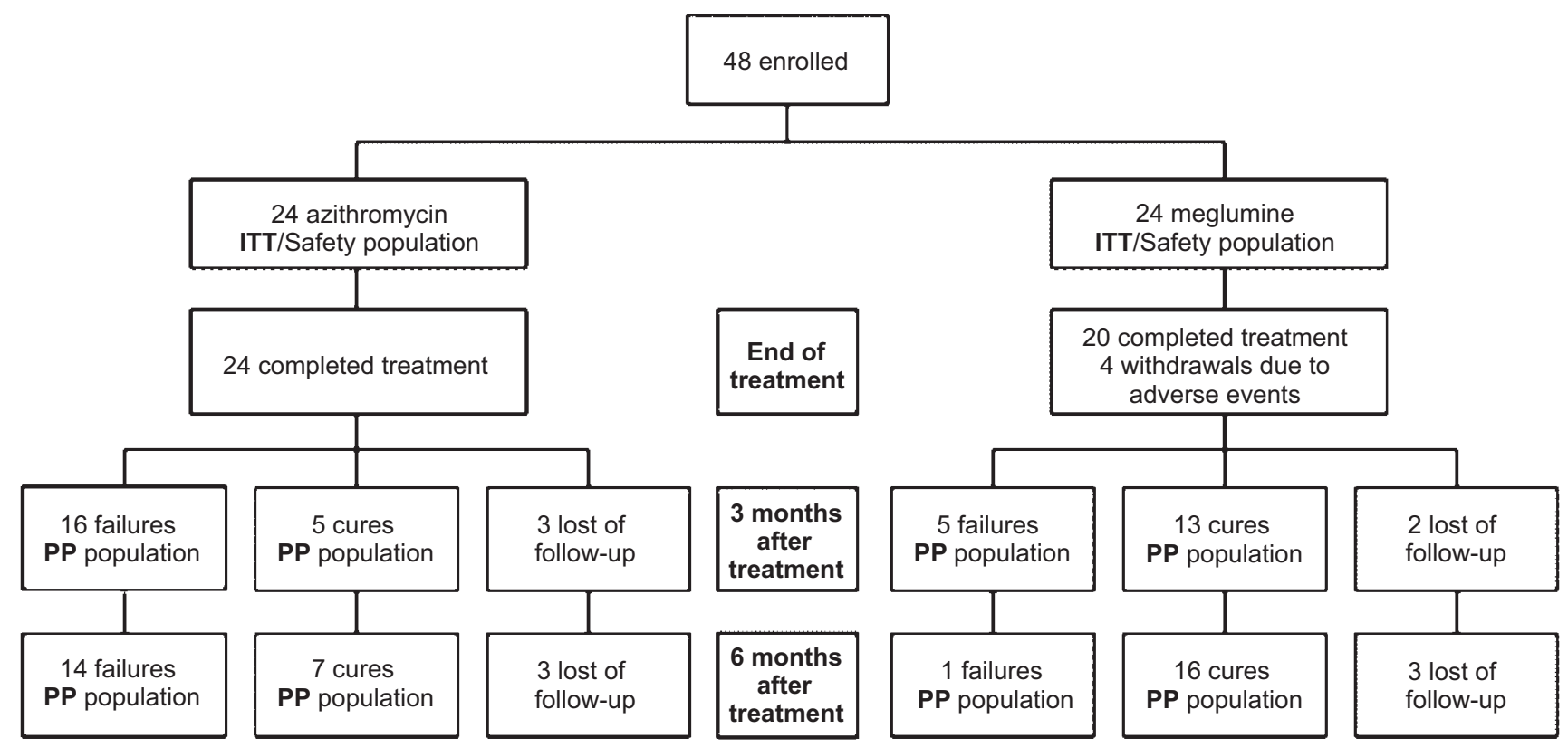

FIGURE 1 - Enrollment, treatment, and follow-up of the study subjects. ITT: intention-to-treat; PP: per protocol.

$1.13-3.42$ ] and $72.2 \%$ versus $23.8 \%$ [RR 3.03 ; 95\% CI $1.34-$ 6.87], respectively). Similar results were observed with the late cure rate (6 months after the end of the treatments). Four cured patients were lost to follow-up between 3 and 6 months after treatments. No clinical recurrence was documented among the patients who effectively returned for their last follow-up visit.

As shown in Table 3, considering the cured patients, despite the the higher percentage of healed lesions when the treatment was completed and 30 days after treatment completion in the meglumine antimoniate group, there were no significant differences between the groups regarding healing speed.
No unexpected adverse events (AE) were observed during the study. A total of 24 patients reported 62 AEs that were possibly related or related with the study drugs, $[15(62.5 \%)$ in the meglumine antimoniate group and nine $(37.5 \%)$ in the azithromycin group ( $\mathrm{p}=0.039$; $\mathrm{RR}=2.188-95 \%$ CI 1.040 4.603)]. The AEs were more frequent in the meglumine antimoniate group (49 events) compared with the azithromycin group (13 events). Regarding severity, the majority (51/62; $82.5 \%$ ) of the AEs were considered mild; however, nine (14.5\%) were moderate and two (3.2\%) were severe (e.g., malaise and vomiting). There were no significant differences between the 
TABLE 2 - Primary and secondary study endpoints.

\begin{tabular}{|c|c|c|c|c|c|c|}
\hline \multirow[b]{2}{*}{ Endpoint } & \multicolumn{2}{|c|}{$\begin{array}{c}\text { Meglumine antimoniate } \\
\text { Group A }\end{array}$} & \multicolumn{2}{|c|}{$\begin{array}{l}\text { Azithromycin } \\
\text { Group B }\end{array}$} & \multirow[b]{2}{*}{ p-value* } & \multirow[b]{2}{*}{$\mathrm{RR}(95 \% \mathrm{CI})$} \\
\hline & $\mathrm{n}$ & $\%$ & $\mathrm{n}$ & $\%$ & & \\
\hline Cure 3 months after treatment (ITT) & $13 / 24$ & 54.2 & $5 / 24$ & 20.8 & 0.036 & $1.97(1.13-3.42)$ \\
\hline Cure 3 months after treatment (PP) & $13 / 18$ & 72.2 & $5 / 21$ & 23.8 & 0.004 & $3.03(1.34-6.87)$ \\
\hline
\end{tabular}

RR: relative risk; 95\% CI: 95\% confidence interval; ITT: intention-to-treat; PP: per protocol. *Fisher's exact test (2-sided).

TABLE 3 - The percentage of healed lesions at treatment completion and at days 30, 60 and 90 after treatment completion.

\begin{tabular}{|c|c|c|c|c|c|}
\hline \multirow[b]{3}{*}{ Time } & \multirow{2}{*}{\multicolumn{2}{|c|}{$\begin{array}{c}\text { Meglumine antimoniate }(\mathrm{n}=13) \\
\text { Group A }\end{array}$}} & \multirow{2}{*}{\multicolumn{2}{|c|}{$\begin{array}{c}\text { Azithromycin }(\mathrm{n}=5) \\
\text { Group B }\end{array}$}} & \multirow[b]{3}{*}{$\mathrm{p}^{*}$} \\
\hline & & & & & \\
\hline & $\mathrm{n}$ & $\%$ & $\mathrm{n}$ & $\%$ & \\
\hline End of treatment & 1 & 7.7 & 0 & 0.0 & $<0.001$ \\
\hline 30 days post-treatment & 8 & 61.5 & 1 & 20.0 & $>0.05$ \\
\hline 90 days post-treatment & 13 & 100.0 & 5 & 100.0 & $>0.05$ \\
\hline
\end{tabular}

*Fisher's exact test (2-sided).

groups. No life threatening conditions were observed during the treatments. The AEs were diverse between the groups (Table 4). The most frequent AEs in the meglumine antimoniate group were myalgia (22.4\%), arthralgia (18.4\%), and malaise $(8.1 \%)$. In the azithromycin group, they were diarrhea $(30.8 \%)$ and abdominal pain (23.1\%).

\section{DISCUSSION}

Until now, few studies have tested azithromycin as an alternative treatment for $\mathrm{CL}^{10-12,14,15}$. Only one study was a randomized clinical trial that compared oral azithromycin and meglumine antimoniate for the treatment of CL in Americas, which included 23 patients in each study group. The authors concluded that azithromycin had a moderate efficacy against L. (V.) braziliensis $(45.5 \%)$ and suggested that a subset of patients could benefit from this therapeutic option ${ }^{10}$.

Nonetheless, a number of reasons supported the decision to compare oral azithromycin and meglumine antimoniate in Brazil and to design a larger randomized non-inferiority trial to test a cure rate difference of not less than $-10 \%$ that could allow the registration of a new use for azithromycin by the Brazilian regulatory agency [Agência Nacional de Vigilância Sanitária (ANVISA)]. It is a consensus that new treatments are urgently needed to improve adherence, cost, cure rates, safety and quality of life. Additionally, there is a concern regarding antimoniate resistance and the need of a safer and better-tolerated treatment ${ }^{20,21}$. Azithromycin is an oral and welltolerated drug with the important advantage of low cost, and oral treatments could improve access and adherence. Moreover, if a moderate efficacy was to be proven, further analysis could identify subsets of patients to whom an oral, less expensive and safer drug could be first offered.

Unfortunately, the high clinical failure rate of azithromycin led to the premature interruption of this study. Even with the small number of patients included until the study interruption, the analysis was able to show the inferiority of azithromycin compared with meglumine antimoniate in the ITT and PP analyses.

The dosing regimen for azithromycin was chosen to allow the comparison with the standard schedule of meglumine antimoniate at a dose with predictable good tolerance for the 20 days treatment, a longer duration than the usual prescription for bacterial infections. Krolewiecki et al. ${ }^{10}$ used azithromycin for 28 days: one gram in the first day and 500mg for the following 27 days. A second cycle at the same doses and lasting 15 days was indicated for patients with clinical improvement without resolution 14 days after completing the first cycle. This dosing difference and the geographical parasite and/or host specificities may have contributed for the slight higher cure rate observed by those authors compared with our observed cure rate $(45.5 \%$ vs. $33.3 \%)^{10}$. 
TABLE 4 - Adverse events related or possibly related to the study drugs.

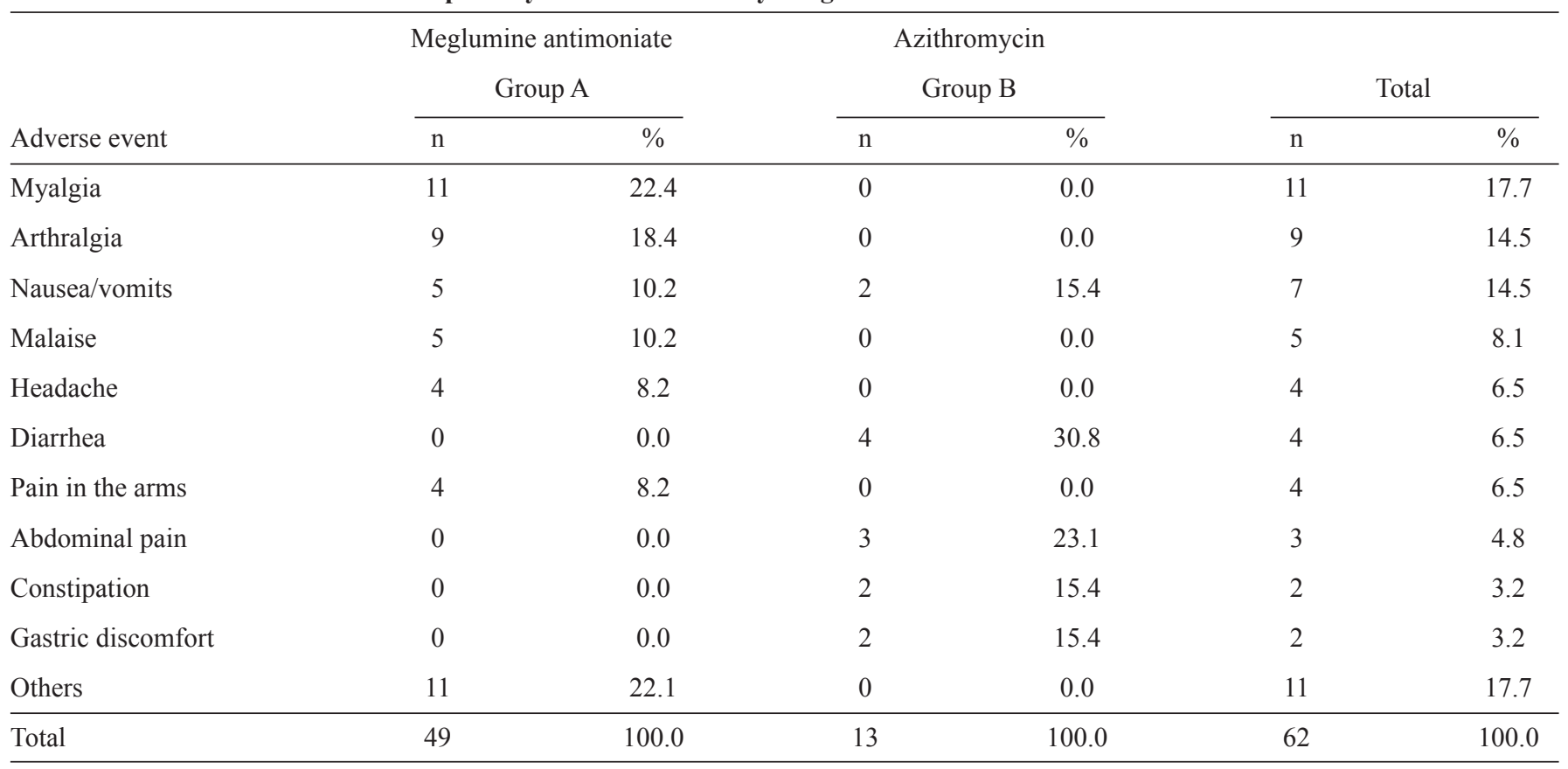

The number of patients presenting AEs and the frequency of those effects were higher in the group treated with meglumine antimoniate, although the severity grade was defined as mild for the large majority in both groups. In the azithromycin group, there were a predominance of gastrointestinal AEs and more diverse systemic effects were observed in the meglumine antimoniate group.

In conclusion, the present study showed that azithromycin is not effective in CL treatment in an area where L. braziliensis is the predominant parasitic species and does not seem to have a role in the therapeutic arsenal for CL in this particular setting. A careful evaluation should be mandatory for future decision making on the need of new clinical trials using azithromycin alone or in combination with other drugs for CL.

\section{ACKNOWLEDGMENTS}

We thank Rosiane Estéfane da Silva, Andréia Coutinho de Faria, Maria Camilo Ribeiro Senna, Tatiana Camilo Ribeiro de Senna and Letícia de Melo Mota for collaborating on this project and for the technical assistance.

\section{CONFLICT OF INTEREST}

The authors declare that there is no conflict of interest.

\section{FINANCIAL SUPPORT}

This work was supported by Conselho Nacional de Desenvolvimento Científico e Tecnológico (CNPq), Fundação de Amparo à Pesquisa do Estado de Minas Gerais (FAPEMIG) and Fundação Oswaldo Cruz (FIOCRUZ). The funders had no role in study design, data collection and analysis, decision to publish, or preparation of the manuscript.

\section{REFERENCES}

1. Alvar J, Vélez ID, Bern C, Herrero M, Desjeux P, Cano J, et al. Leishmaniasis worldwide and global estimates of its incidence. PLoS One 2012; 7:e35671.

2. Ministério da Saúde. Sistema de Informação de Agravos de Notificação (Sinan Net). Reported cases of American Tegumentary Leishmaniasis [Internet]. Ministério da Saúde; 2013. [Cited 2014 May 13]. Available at: http://dtr2004.saude.gov.br/sinanweb/tabnet/ dh?sinannet/lta/bases/ltabrnet.def/.

3. Murray HW, Berman JD, Davies CR, Saravia NG. Advances in leishmaniasis. Lancet 2005; 366:1561-1577.

4. Toledo Jr AC, da Silva RE, Carmo RF, Amaral TA, Luz ZM, Rabello A. Assessment of the quality of life of patients with cutaneous leishmaniasis in Belo Horizonte, Brazil, 2009-2010. A pilot study. Trans R Soc Trop Med Hyg 2013; 107:335-336.

5. Machado PR, Penna G. Miltefosine and cutaneous leishmaniasis. Curr Opin Infect Dis 2012; 25:141-144.

6. Soto J, Soto P. Miltefosine: oral treatment of leishmaniasis. Expert Rev Anti Infect Ther 2006; 4:177-185.

7. Soto J, Toledo J, Gutierrez P, Nicholls RS, Padilla J, Engel J, et al. Treatment of American cutaneous leishmaniasis with miltefosine, an oral agent. Clin Infect Dis 2001; 33:E57-61.

8. Alrajhi AA, Ibrahim EA, De Vol EB, Khairat M, Faris RM, Maguire JH. Fluconazole for the treatment of cutaneous leishmaniasis caused by Leishmania major. N Engl J Med 2002; 346:891-895.

9. Sousa AQ, Frutuoso MS, Moraes EA, Pearson RD, Pompeu MM. High-dose oral fluconazole therapy effective for cutaneous leishmaniasis due to Leishmania (Vianna) braziliensis. Clin Infect Dis 2011; 53:693-695. 
10. Krolewiecki AJ, Romero HD, Cajal SP, Abraham D, Mimori T, Matsumoto $\mathrm{T}$, et al. A randomized clinical trial comparing oral azithromycin and meglumine antimoniate for the treatment of American cutaneous leishmaniasis caused by Leishmania (Viannia) braziliensis. Am J Trop Med Hyg 2007; 77:640-646.

11. Layegh P, Yazdanpanah MJ, Vosugh EM, Pezeshkpoor F, Shakeri MT, Moghiman T. Efficacy of azithromycin versus systemic meglumine antimoniate (Glucantime) in the treatment of cutaneous leishmaniasis. Am J Trop Med Hyg 2007; 77:99-101.

12. Momeni AZ, Shafiei A, Emamjoeh M, Aminjavaheri M, Momeni A. Azithromycin is not effective in the treatment of old world cutaneous leishmaniasis. Eur J Dermatol 2006; 16:701-702.

13. Prata A, Silva-Vergara ML, Costa L, Rocha A, Krolewiecki A, Silva JC, et al. Efficacy of azithromycin in the treatment of cutaneous leishmaniasis. Rev Soc Bras Med Trop 2003; 36:65-69.

14. Teixeira AC, Paes MG, Guerra JO, Prata A, Silva-Vergara ML. Low efficacy of azithromycin to treat cutaneous leishmaniasis in Manaus, AM, Brazil. Rev Inst Med Trop Sao Paulo 2007; 49:235-238.

15. Teixeira AC, Paes MG, Guerra JO, Prata A, Silva-Vergara ML. Failure of both azithromycin and antimony to treat cutaneous leishmaniasis in Manaus, AM, Brazil. Rev Inst Med Trop Sao Paulo 2008; 50:157-160.
16. Oliveira-Silva F, Morais-Teixeira E, Rabello A. Antileishmanial activity of azithromycin against Leishmania (Leishmania) amazonensis, Leishmania (Viannia) braziliensis, and Leishmania (Leishmania) chagasi. Am J Trop Med Hyg 2008; 78:745-749.

17. Krolewiecki A, Leon S, Scott P, Abraham D. Activity of azithromycin against Leishmania major in vitro and in vivo. Am J Trop Med Hyg 2002; 67:273-277.

18. Tanyuksel M, Bas AL, Araz E, Aybay C. Determination of intracellular efficacies of azithromycin against Leishmania major infection in human neutrophils in vitro. Cell Biochem Funct 2003; 21:93-96.

19. Passos VM, Fernandes O, Lacerda PA, Volpini AC, Gontijo CM, Degrave W, et al. Leishmania (Viannia) braziliensis is the predominant species infecting patients with American cutaneous leishmaniasis in the State of Minas Gerais, Southeast Brazil. Acta Trop 1999; 72:251-258.

20. Wordl Health Organization (WHO). Department of control of neglected tropical diseases. Sustaining the drive to overcome the global impact of neglected tropical diseases. Second WHO report on neglected tropical diseases. Geneve: WHO; 2013.

21. Croft SL, Sundar S, Fairlamb AH. Drug resistance in leishmaniasis. Clin Microbiol Rev 2006; 19:111-126. 